\title{
Regressive Structures for Computation of DST-II and Its Inverse
}

\author{
Priyanka Jain ${ }^{1}$ and Anamika Jain ${ }^{2}$ \\ ${ }^{1}$ Department of Electronics and Communication, Delhi Technological University, Shahbad Doulatpur, \\ Main Bawana Road, Delhi-110042, India \\ ${ }^{2}$ Department of Electronics and Communication, 245 Budhpur, GT Karnal Road, Industrial Area, \\ National Highway 1, Delhi 110036, India
}

Correspondence should be addressed to Priyanka Jain, priyajain2000@rediffmail.com

Received 31 August 2012; Accepted 14 October 2012

Academic Editors: P. Corsonello and J. Solsona

Copyright () 2012 P. Jain and A. Jain. This is an open access article distributed under the Creative Commons Attribution License, which permits unrestricted use, distribution, and reproduction in any medium, provided the original work is properly cited.

Efficient regressive structures for implementation of forward (DST-II) and inverse discrete sine transform (IDST-II) are developed. The proposed algorithm not only minimizes the arithmetic complexity compared to the existing algorithms (Wany (1990), Hupta and Rao (1990), Yip and Rao (1987), Murthy and Swamy (1992)) but also provides hardware savings over the algorithm (Jain et al. (2008)) by the same authors. The naturally ordered input sequence makes the new algorithms suitable for on-line computation.

\section{Introduction}

Discrete sine transform (DST) and discrete cosine transform (DCT) are used as key functions in many signal and image processing applications, for example, block filtering, transform domain adaptive filtering, digital signal interpolation, adaptive beam forming, image resizing, speech enhancement [1-6] and so forth, due to their near optimal transform coding performance. Both DST and DCT are good approximations to the statistically optimal Karhunen-Loeve transform $[7,8]$. It is found that in case of image with high correlation coefficient, DCT-based coding results in better performance but for low correlation image, DST gives lower bit rate [8]. Since both DCT and DST are computationally intensive, many efficient algorithms have been proposed to improve the performance of their implementation [9-12]; however most of these are only good software solutions. Chiang and Liu [13] suggested a regressive structure for DCT-IV and DST-IV using second order digital filters. This paper is aimed at developing a similar regressive filter structure for DST-II/IDST-II that provides considerable hardware saving compared to the existing algorithm [14] and also reduces the computation complexity in terms of number of operation (multiplication and addition) as compared to the existing algorithms [15-18]. The advantage of this implementation is that no permutation is required for the input/output sequences, and therefore it is especially suitable for on-line computation.

\section{Regressive Formulas of DST-II/IDST-II and Realization through Filter Structure}

The DST of a sequence $y(n), n=1,2, \ldots, N$ and its inverse are given by [14]

$$
Y[k]=\gamma_{k} \sum_{n=1}^{N} y(n) \sin \left[\left(n-\frac{1}{2}\right) \frac{k \pi}{N}\right], \quad k=1,2, \ldots, N
$$

where

$$
\gamma_{k}=\sqrt{\frac{2}{N}} \varepsilon_{k}, \quad \varepsilon_{k}=\left\{\begin{array}{l}
\frac{1}{\sqrt{2}} \quad k=N \\
1 \quad k=1,2, \ldots, N-1,
\end{array}\right.
$$

and

$$
\begin{array}{r}
y(n)=\sqrt{\frac{2}{N}} \sum_{k=1}^{N} \varepsilon_{k} Y[k] \sin \left[\left(n-\frac{1}{2}\right) \frac{k \pi}{N}\right], \\
n=1,2, \ldots, N .
\end{array}
$$




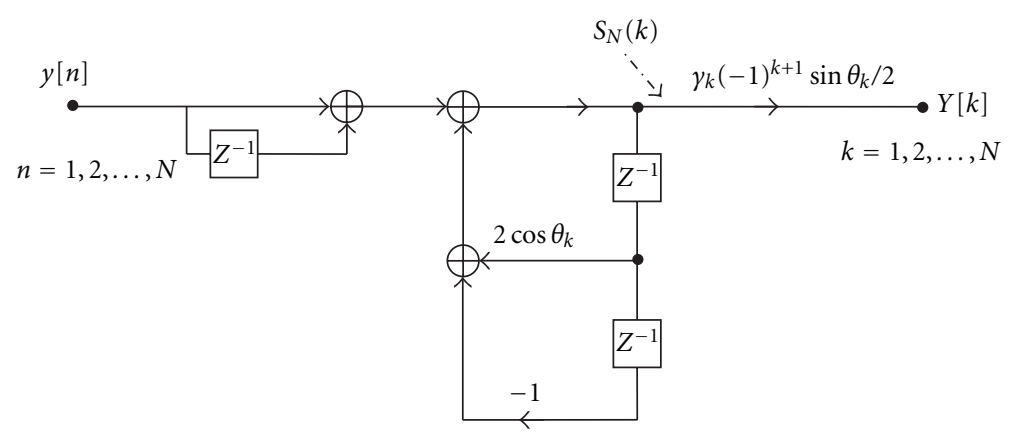

FigURE 1: Regressive structure for computation of DST-II.

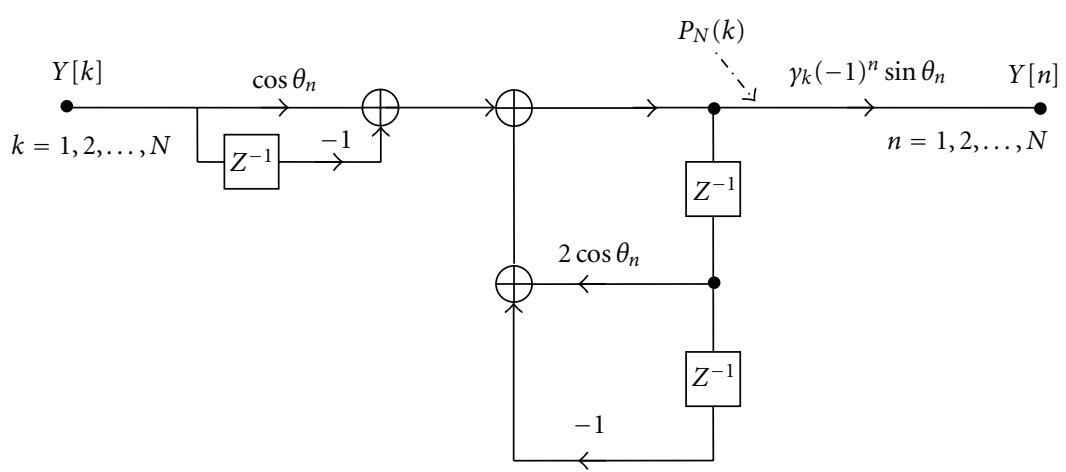

FIgURE 2: Regressive structure for computation of IDST-II.

Replacing $n$ by $N-n$ in (1a), $k$ by $N-k$ in (2), and after some algebraic manipulations, we obtain DST-II/IDST-II in the form

$$
\begin{array}{r}
Y[k]=(-1)^{k+1} \gamma_{k} \sum_{n=0}^{N-1} y(N-n) \sin \left[\left(n+\frac{1}{2}\right) \frac{k \pi}{N}\right], \\
k=1,2, \ldots, N \\
y(n)=(-1)^{n} \sqrt{\frac{2}{N}} \sum_{k=0}^{N-1} \varepsilon_{k} Y[N-k] \cos \left[\left(n-\frac{1}{2}\right) \frac{k \pi}{N}\right], \\
n=1,2, \ldots, N .
\end{array}
$$

Let $\theta_{k}=k \pi / N ; \theta_{n}=(n-1 / 2)(\pi / N)$ and $x_{n}=\cos \theta_{n}$.

Further we define

$$
\begin{gathered}
V_{n}\left[x_{k}\right]=\frac{\sin (n+1 / 2) \theta_{k}}{\sin \left(\theta_{k} / 2\right)}, \quad k=1,2, \ldots, N, \\
U_{k}\left[x_{n}\right]=\frac{\cos k \theta_{n}}{\cos \theta_{n}}, \quad n=1,2, \ldots, N .
\end{gathered}
$$

We may rewrite (3) in the form

$$
\begin{array}{r}
Y[k]=(-1)^{k+1} \sin \frac{\theta_{k}}{2} \gamma_{k} \sum_{n=0}^{N-1} y(N-n) V_{k}\left[x_{k}\right], \\
k=1,2, \ldots, N,
\end{array}
$$

$$
\begin{array}{r}
y[n]=(-1)^{n} \cos \theta_{n} \sqrt{\frac{2}{N}} \sum_{k=0}^{N-1} \varepsilon_{k} Y[N-k] U_{k}\left[x_{n}\right], \\
n=1,2, \ldots, N .
\end{array}
$$

Further, we can write $V_{n+1}\left[x_{k}\right]$ as

$$
\begin{array}{r}
V_{n+1}\left[x_{k}\right]=\frac{\sin (n+3 / 2) \theta_{k}}{\sin \left(\theta_{k} / 2\right)}, \\
k=1,2, \ldots, N,
\end{array}
$$

or

$$
\begin{array}{r}
V_{n+1}\left[x_{k}\right]=\frac{2 \sin (n+1 / 2) \theta_{k} \cos \theta_{k}-\sin (n-1 / 2) \theta_{k}}{\sin \left(\theta_{k} / 2\right)}, \\
k=1,2, \ldots, N .
\end{array}
$$

Equation (9) can be represented by a sinusoidal recursive formula as

$$
V_{n+1}\left[x_{k}\right]=2 \cos \theta_{k} V_{n}-V_{n-1}, \quad k=1,2, \ldots, N
$$

In the same manner, we derive the sinusoidal formula for (5) as

$$
U_{k+1}\left[x_{n}\right]=2 \cos \theta_{k} U_{k}-U_{k-1}, \quad n=1,2, \ldots, N
$$


In (6) and (7), we define

$$
\begin{aligned}
S_{N}(k) & =\sum_{n=0}^{N-1} y(N-n) V_{n}\left[x_{k}\right], \\
P_{N}(k) & =\sum_{k=0}^{N-1} Y[N-k] U_{k}\left[x_{n}\right] .
\end{aligned}
$$

Using the sinusoidal recursive formula given in (10) and the fact that, $V_{0}=1 ; V_{-1}=-1$ and $U_{0}=\cos \theta_{n},(12)$ can be written as

$$
\begin{aligned}
S_{N}(k) & =y(N) V_{0}+\sum_{n=1}^{N-1} y(n-n) V_{n}\left[x_{k}\right] \\
& =y(N)+\sum_{n=0}^{N-2} y(N-n-1) V_{n+1}\left[x_{k}\right] \\
& =y(N)+\sum_{n=0}^{N-2} y(N-n-1)\left[2 \cos \theta_{k} V_{n}-V_{n-1}\right] \\
& =y(N)+2 \cos \theta_{k} S_{N-1}(k)-y(N-1) V_{-1}-S_{N-2}(k) .
\end{aligned}
$$

Similarly, (13) is written as

$$
\begin{aligned}
P_{N}(k) & =Y[N] U_{0}+\sum_{k=1}^{N-1} Y[N-k] U_{k}\left[x_{n}\right] \\
& =\frac{Y[N]}{\cos \theta_{n}}+\sum_{k=0}^{N-2} Y[N-k-1] U_{k+1}\left[x_{n}\right] \\
& =\frac{Y[N]}{\cos \theta_{n}}+2 \cos \theta_{k} P_{N-1}(k)-Y[N-1]-P_{N-2}(k),
\end{aligned}
$$

where $U_{-1}=1$. Using (6) and (12), the DST-II can be expressed as

$$
Y[k]=(-1)^{k+1} \gamma_{k} \sin \frac{\theta_{k}}{2} S_{N}(k), \quad k=1,2, \ldots, N .
$$

Similarly, using (7) and (13), the IDST expression is

$$
y(n)=(-1)^{n} \gamma_{k} \cos \theta_{n} P_{N}(k), \quad n=1,2, \ldots, N .
$$

From (16) and (17), DST-II/IDST-II can be implemented with the second order filter structure shown in Figures 1 and 2.

\section{Performance}

The proposed algorithm has been presented with the aim of realizing the DST/IDST of any length for on-line computation. It can be seen from the regressive structure for DSTII shown in Figure 1 that only one real multiplication per sample is required to bring the system in a state from which $S_{N}(k)$ can be computed. Similarly the regressive structure
TABLE 1: Hardware comparison for implementing DST-II/IDST-II coefficient.

\begin{tabular}{lccccc}
\hline \multirow{2}{*}{ S. No. } & \multirow{2}{*}{ Comparison } & \multicolumn{2}{c}{ Proposed } & \multicolumn{2}{c}{$[14]$} \\
& & DST & IDST & DST & IDST \\
\hline 1 & Latches & 2 & 2 & 4 & 4 \\
2 & Real multiplier & 2 & 3 & 4 & 4 \\
3 & Adder & 3 & 3 & 6 & 8 \\
4 & Complex multiplier & 0 & 0 & 0 & 0 \\
5 & Computation cycles & $N$ & $N$ & $N / 2$ & $N / 2$ \\
\hline
\end{tabular}

TABLE 2: Comparison of algorithms for computing DST in terms of addition and multiplication.

\begin{tabular}{lcc}
\hline \multirow{2}{*}{ Algorithms } & $\begin{array}{c}\text { Number of addition } \\
\end{array}$ & $\begin{array}{c}\text { Number of multiplication } \\
N=2^{M}\end{array}$ \\
\hline Fast DST, [15, 16] & $3 M N / 2-N+1$ & $M N / 2+1$ \\
{$[17]$} & $4 N+2$ & $6 N$ \\
{$[18]$} & $5 N+2$ & $5 N$ \\
The proposed design & $3 N$ & $(N+1)$ \\
\hline
\end{tabular}

shown in Figure 2 for IDST shows that two real multiplications are required for $P_{N}(k)$ computation. The constant multiplication by $(-1)^{k+1} \gamma_{k} \sin \left(\theta_{k} / 2\right)$ or $(-1)^{n} \gamma_{k} \cos \theta_{n}$ at the output need not be performed at every iteration of the difference equation but it is to be multiplied after the Nth step. Hence the total real multiplications for DST computation are $(N+1)$ and for IDST computation are $(2 N+1)$. The number of additions for DTS as well as for IDST is $3 N$. In Table 1 the number of multipliers, adders and latches required by the present algorithm is compared with those required in algorithms [14]. It can be seen from Table 1 that the new recursive algorithm is efficient in terms of saving the number of multipliers, adder, and latches compared to algorithm [14]. Both of the algorithms compute the DST/IDST of an $N$ point real sequence. Although the proposed algorithm does not optimize the number of computational cycle, but has advantage in terms of hardware compared to previous approach [14]. Further, the filter structure is numerically stable, as it involves no division at all. Algorithm [14] needs addition and subtraction of input sequence whereas the input sequence for the present algorithm is in the natural order, which makes the proposed approach suitable for on-line computation. Table 2 shows the comparison of computational complexity of the proposed algorithm with the other algorithms [15-18]. It can be seen that the proposed algorithm not only reduces the number of multiplication significantly but also reduces the total number of operations.

\section{Conclusion}

The proposed regressive algorithm has been derived mathematically and realized for kernels of DST and its inverse. This algorithm is effective for realization using software and hardware techniques. This algorithm provides substantial savings in actual number of multipliers, adders, and latches 
in the hardware implementation required to perform the DST/IDST and reduces the arithmetic complexity compared to the existing algorithms. The proposed regressive algorithm has simple, regular, and modular filter structure and is particularly suitable for on-line computation.

\section{Acknowledgment}

The authors would like to thank the associate editor and referees for their valuable comments to improve the paper.

\section{References}

[1] Z. Wang, G. A. Jullien, and W. C. Miller, "Interpolation using the discrete sine transform with increased accuracy," Electronics Letters, vol. 29, no. 22, pp. 1918-1920, 1993.

[2] S. A. Martucci and R. M. Mersereau, "New approaches to block filtering of images using symmetric convolution and the DST or DCT," in Proceedings of IEEE International Symposium on Circuits and Systems (ISCAS '93), pp. 259-262, May 1993.

[3] F. Beaufays, "Transform-domain adaptive filters: an analytical approach," IEEE Transactions on Signal Processing, vol. 43, no. 2, pp. 422-431, 1995.

[4] Y. S. Park and H. W. Park, "Arbitrary-ratio image resizing using fast DCT of composite length for DCT-based transcoder," IEEE Transactions on Image Processing, vol. 15, no. 2, pp. 494-500, 2006.

[5] L. Xueyao, X. Hua, and C. Bailing, "Noisy speech enhancement based on discrete sine transform," in Proceedings of the 1st International Multi-Symposiums on Computer and Computational Sciences (IMSCCS '06), pp. 199-202, April 2006.

[6] S. C. Lim, D. Y. Kim, and Y. L. Lee, "Alternative transform based on the correlation of the residual signal," in Proceedings of the 1st International Congress on Image and Signal Processing (CISP '08), pp. 389-394, May 2008.

[7] R. J. Clarke, "Relation between the Karhunen Loeve and Sine transform," Electronics Letters, vol. 20, no. 1, pp. 12-13, 1984.

[8] A. K. Jain, "Fast Karhunen-Loeve transform for a class of stochastic processes," IEEE Transactions on Communications, vol. 24, no. 9, pp. 1023-1029, 1976.

[9] V. Kober, "Fast algorithms for the computation of sliding discrete sinusoidal transforms," IEEE Transactions on Signal Processing, vol. 52, no. 6, pp. 1704-1710, 2004.

[10] D. Püschel and J. M. F. Moura, "Algebraic signal processing theory: cooley-Tukey type algorithms for DCTs and DSTs," IEEE Transactions on Signal Processing, vol. 56, no. 4, pp. 15021521, 2008.

[11] P. K. Meher and M. N. S. Swamy, "New systolic algorithm and array architecture for prime-length discrete sine transform," IEEE Transactions on Circuits and Systems II, vol. 54, no. 3, pp. 262-266, 2007.

[12] P. K. Meher, A. P. Vinod, J. C. Patra, and M. N. S. Swamy, "Reduced-complexity concurrent systolic implementation of the discrete sine transform," in Proceedings of IEEE Asia Pacific Conference on Circuits and Systems (APCCAS '06), pp. 15351538, December 2006.

[13] H. C. Chiang and J. C. Liu, "A regressive structure for online computation of arbitrary length DCT-IV and DST-IV transforms," IEEE Transactions on Circuits and Systems for Video Technology, vol. 6, no. 6, pp. 692-695, 1996.

[14] P. Jain, B. Kumar, and S. B. Jain, "Discrete sine transform and its inverse-realization through recursive algorithms,"
International Journal of Circuit Theory and Applications, vol. 36, no. 4, pp. 441-449, 2008.

[15] Z. Wang, "Fast discrete sine transform algorithms," Signal Processing, vol. 19, no. 2, pp. 91-102, 1990.

[16] A. Gupta and K. R. Rao, "Fast recursive algorithm for the discrete sine transform," IEEE Transactions on Acoustics, Speech, and Signal Processing, vol. 38, no. 3, pp. 553-557, 1990.

[17] P. Yip and K. R. Rao, "On the shift properties of DCT's and DST's," IEEE Transactions on Acoustics, Speech, and Signal Processing, vol. 35, no. 3, pp. 404-406, 1987.

[18] N. R. Murthy and M. N. S. Swamy, "On the computation of running discrete cosine and sine transform," IEEE Transactions on Signal Processing, vol. 40, no. 6, pp. 1430-1437, 1992. 

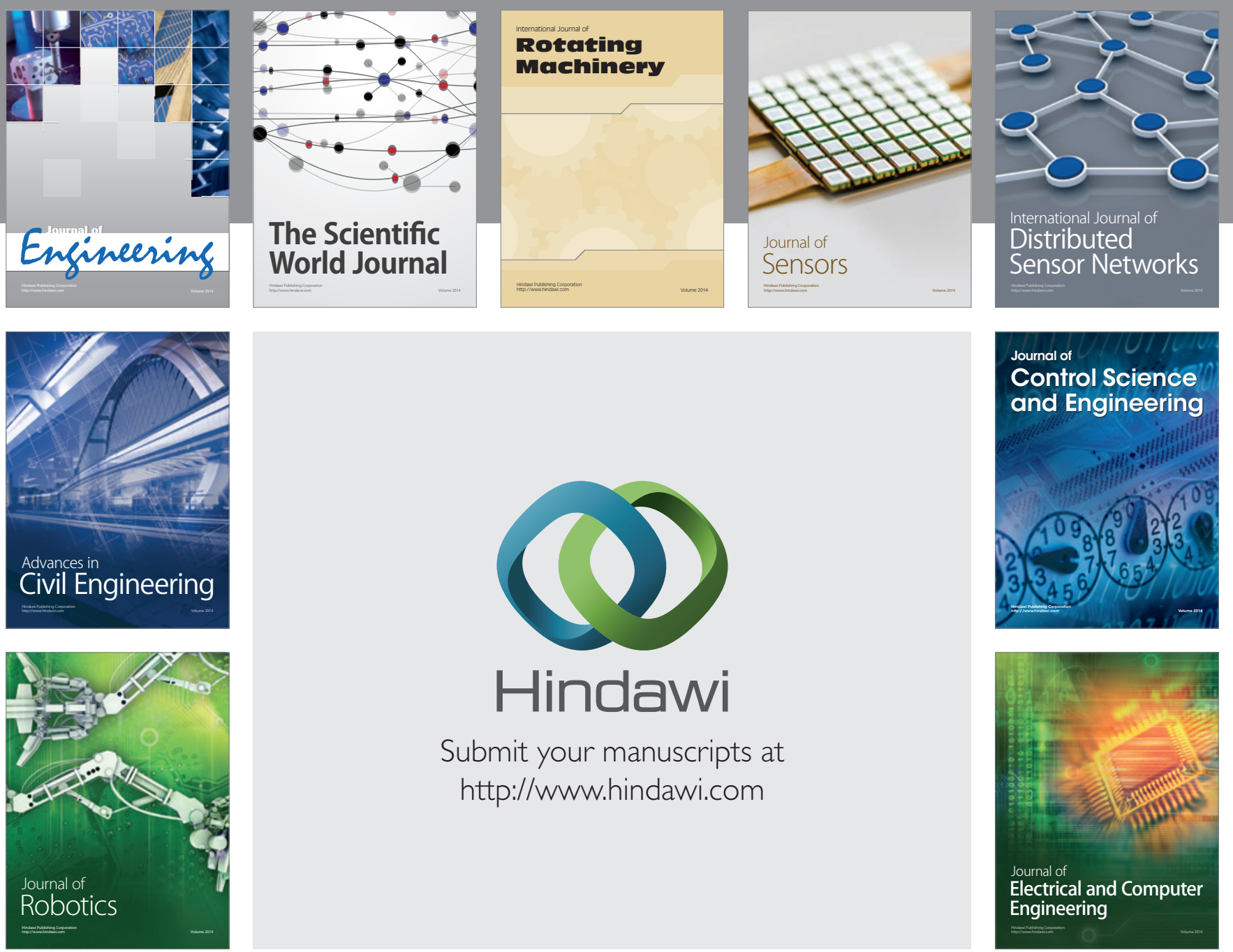

Submit your manuscripts at

http://www.hindawi.com
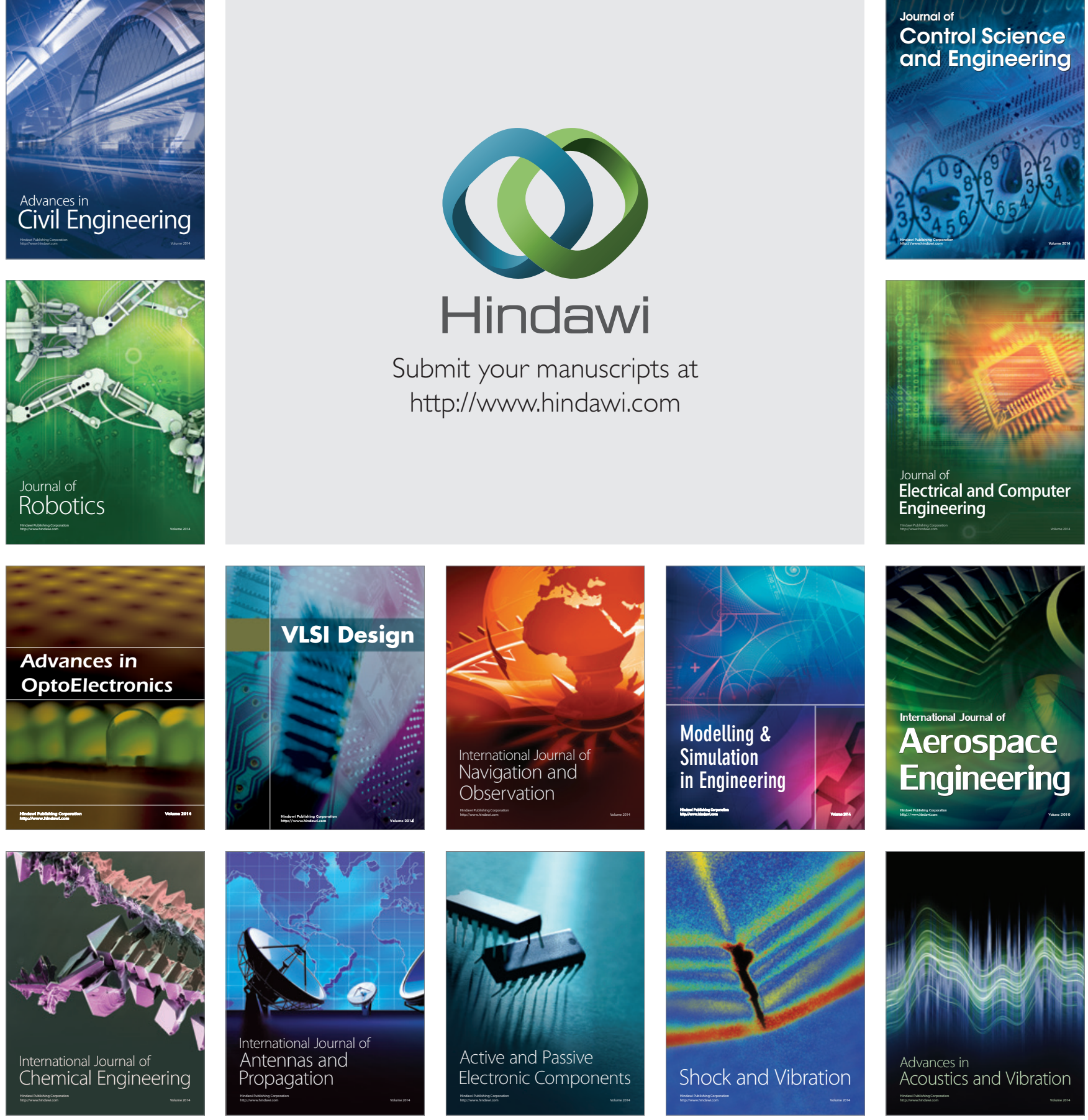